\title{
Aloperine attenuates high glucose-induced oxidative injury in Schwann cells via activation of NRF2/HO-1 pathway
}

\author{
Yiran Chen ${ }^{1}$, Tieming $\mathrm{Ma}^{1 *}$, Zhimin $\mathrm{Wang}^{2}$, Lianqun $\mathrm{Jia}^{3}$, Xiaoqing Zhang ${ }^{1}$, \\ Qingxuan $\mathrm{He}^{1}$, Sijia Liu ${ }^{1}$ \\ ${ }^{1}$ College of Acupuncture and Massage, Liaoning University of Traditional Chinese Medicine, ${ }^{2}$ Department of Endocrinology, \\ Affiliated Hospital of Liaoning University of Traditional Chinese Medicine, ${ }^{3}$ Key Laboratory of Ministry of Education for \\ Traditional Chinese Medicine, Viscera-State Theory and Applications, Liaoning University of Traditional Chinese Medicine, \\ Shenyang City, Liaoning Province 110000, China
}

*For correspondence: Email: TiemingMadkl@163.com; Tel: +86-24 31207131

Sent for review: 10 March 2020

Revised accepted: 26 May 2020

\begin{abstract}
Purpose: To determine the involvement of nuclear factor erythroid 2-related factor 2 (NRF2) and heme oxygenase-1 (HO-1) in the action of aloperine on Schwann cell injury caused by high glucose (HG). Methods: Cell viability was determined using MTT assay while the release of lactate dehydrogenase $(\mathrm{LDH})$ was determined by biochemical assay. Apoptosis was assessed using flow cytometry, while the levels of malondialdehyde (MDA) were determined by Annexin V-FIT staining. Glutathione Stransferase (GST), glutathione peroxidase (GPX), and reactive oxygen species (ROS) were determined using enzyme-linked immunosorbent assay.

Results: Treatment with HG suppressed RSC96 cell viability and increased LDH release, while aloperine reversed these results $(p<0.05)$. Apoptosis of RSC96 cells was induced by HG stimulation, but was abolished by aloperine. The levels of ROS, MDA, and GST were enhanced in cells following treatment with HG, but was reversed by aloperine $(p<0.05)$. The decreased level of GPX caused by HG in RSC96 cells was elevated by aloperine. Moreover, aloperine upregulated NRF2 and HO-1 in RSC96 cells treated with HG ( $p<0.05)$.

Conclusion: Aloperine attenuates HG-induced oxidative injury in Schwann cells via activation of NRF2/HO-1 pathway, suggesting its potential as a potent drug for the management of diabetic peripheral neuropathy.
\end{abstract}

Keywords: Aloperine, Schwann cells, High glucose, Oxidative stress, NRF2, HO-1

\begin{abstract}
This is an Open Access article that uses a fund-ing model which does not charge readers or their institutions for access and distributed under the terms of the Creative Commons Attribution License (http://creativecommons.org/licenses/by/4.0) and the Budapest Open Access Initiative (http://www.budapestopenaccessinitiative.org/read), which permit unrestricted use, distribution, and
\end{abstract} reproduction in any medium, provided the original work is properly credited.

Tropical Journal of Pharmaceutical Research is indexed by Science Citation Index (SciSearch), Scopus, International Pharmaceutical Abstract, Chemical Abstracts, Embase, Index Copernicus, EBSCO, African Index Medicus, JournalSeek, Journal Citation Reports/Science Edition, Directory of Open Access Journals (DOAJ), African Journal Online, Bioline International, Open-J-Gate and Pharmacy Abstracts

\section{INTRODUCTION}

Diabetic peripheral neuropathy (DPN) is considered a common complication of diabetes mellitus (DM, type 2), which is characterized by peripheral nerve dysfunction [1,2]. The symptoms of DPN include pain, paresthesia, and neurological deficits [3]. Schwann cells exert a vital role in peripheral nerves [4], which are also sensitive to hyperglycemia, and the injury in Schwann cells induced by oxidative stress under long-term hyperglycemia contributes to DPN [5]. Schwan cell damage caused by high glucose (HG) may affect nerve conduction velocity, cause 
atrophy of axons, and alter axonal regeneration [6]. Thus, it is essential to identify the underlying mechanism of Schwann cell injury caused by HG and to develop promising therapeutic strategies for DPN treatment and prevention.

Oxidative stress is a critical mechanism for HGinduced Schwan cell injury [7]. Some studies have suggested that the nuclear factor erythroid 2-related factor 2 (NRF2)/heme oxygenase-1 $(\mathrm{HO}-1)$ pathway is involved in the modulation of oxidative responses [8-10]. In retinal pigment epithelial cells, salvianolic acid A can prevent oxidative stress through activation of the NRF2/HO-1 pathway [8]. This signaling pathway also mediates the HG-induced Schwan cell injury [11], and also participates in the regulatory process of Schwan cell injury induced by $\mathrm{HG}$ [11]. Therefore, this signaling pathway is essential for alleviating oxidative injury in Schwann cells.

Aloperine is an alkaloid extracted from Sophora alopecuroides [12]. Aloperine has multiple functions including anti-tumor, anti-inflammatory, and antiallergenic activities in some diseases $[12,13]$. In addition, aloperine also modulates oxidative stress [14]. $\mathrm{Xu}$ et al reported that aloperine alleviated neuropathic pain by suppressing oxidative stress and NF-kB pathway activation [14].

In a subarachnoid hemorrhage model, aloperine attenuated brain injury by inhibiting oxidative stress through activating the NRF2/HO-1 pathway [15]. Therefore, aloperine may reduce oxidative injury of Schwann cells caused by HG via the NRF2/HO-1 pathway.

Thus, the objective of this study was to investigate the influence of aloperine on the oxidative injury of Schwann cells caused by HG, and to determine the involvement of the NRF2/HO-1 pathway.

\section{EXPERIMENTAL}

\section{Cell culture}

RSC96 Schwann cells were obtained from the American Type Culture Collection (Manassas, VA, USA) and maintained in DMEM medium with fetal bovine serum (FBS; 10\%; Gibco, Grand Island, NY, USA) in an incubator (5\% $\mathrm{CO}_{2}$; $37^{\circ} \mathrm{C}$ ). The cells were cultured for $12 \mathrm{~h}$ and were co-treated with $25 \mathrm{mM}$ (low glucose) or $150 \mathrm{mM}$ glucose (high glucose; $\mathrm{HG}$ ) and 1, 10, or $50 \mu \mathrm{M}$ aloperine. The chemical formula of aloperine is shown in Figure $1 \mathrm{~A}$.

\section{Cell viability assay}

Cells after co-treatments with glucose and aloperine were inoculated into 96-well plates (3x $10^{3}$ cells/well) for $24 \mathrm{~h}$ and added with $20 \mu \mathrm{L}$ of MTT for another $4 \mathrm{~h}$. Subsequently, the formazan crystals were solubilized by adding $200 \mu \mathrm{L}$ of dimethyl sulfoxide. Absorbance was determined at $490 \mathrm{~nm}$.

\section{Lactate dehydrogenase (LDH) assay}

LDH release from RSC96 cells after cotreatments with glucose and aloperine was measured by an LDH assay kit (Abcam, Cambridge, UK). The absorbance was determined at $490 \mathrm{~nm}$.

\section{Flow cytometry}

RSC96 cells after co-treatments with glucose and aloperine were collected, washed, centrifuged, and re-suspended in binding buffer, and then treated with Annexin V-FITC (5 $\mu \mathrm{L})$, stained with propidium iodide (PI, $5 \mu \mathrm{L})$, and quantitated using flow cytometry (BD Biosciences, San Jose, CA, USA).

\section{qRT-PCR}

Total RNA was isolated, cDNA was produced, and the qRT-PCR was performed using a SYBR Green Master Mix (Thermo Fisher Scientific, Scotts Valley, CA, USA) with the primer sequences for NRF2 [16] and HO-1 [17] (see Table 1 for primer sequences). The $2^{-\Delta \Delta C t}$ method was used to calculate the relative expression levels [18].

\section{Western blotting}

Proteins were extracted, quantified, and the lysates were subjected to SDS-PAGE and transferred to polyvinylidene difluoride membranes. After blocking, the membranes were probed with anti-NRF2 (dilution, 1:500) (Abcam, Cambridge, UK), anti-HO-1 (dilution, 1:500) (Abcam), and anti- $\beta$-actin (dilution, 1:1,000) (Abcam) for $12 \mathrm{~h}$ at $4^{\circ} \mathrm{C}$, and then incubated with anti-IgG secondary antibody (Abcam). The blots were visualized using an ECL chemiluminescence reagent kit (Beyotime, Shanghai, China). The relative protein levels were determined by normalizing to $\beta$-actin.

\section{Enzyme-linked immunosorbent assay (ELISA) and ROS release analyses}

After co-treatment with glucose and aloperine, RSC96 cells were harvested and 
malondialdehyde (MDA), glutathione Stransferase (GST), and glutathione peroxidase (GPX) levels were determined using ELISA assay kits. ROS production was evaluated using a Cellular ROS assay kit (Abcam).

\section{Statistical analysis}

Statistical analysis was conducted using SPSS statistical software (SPSS, Chicago, IL, USA). All the data are shown as mean \pm standard deviation (SD). One-way analysis of variance with the least significant difference post hoc test was used to compare differences among multiple groups. $P<0.05$ was regarded as statistically significant.

\section{RESULTS}

\section{Aloperine alleviated HG-induced RSC96 cell injury}

To investigate the influence of aloperine on HGinduced cell injury, Schwann cells (RSC96) were co-treated with HG and aloperine. Treatment with HG (150 mM) reduced the viability of RSC96 cells compared to cells treated with $25 \mathrm{mM}$ glucose. However, aloperine dose-dependently increased cell viability ( $p<0.05$; Figure $1 \mathrm{~B}$ ). Furthermore, LDH release was induced by HG treatment $(p<0.01)$, but was reversed by aloperine $(p<0.05$; Figure $1 \mathrm{C})$. These results indicated that aloperine alleviated HG-induced RSC96 cell injury.
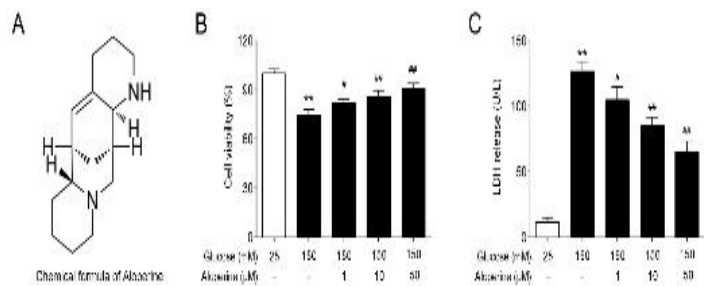

Figure 1: Aloperine reduced RSC96 cell injury induced by high glucose $(150 \mathrm{mM})$. A. The chemical formula of aloperine. B. The cell viability was determined using the MTT assay. C. The lactate dehydrogenase release in different cell groups; ${ }^{* *} p<$ 0.01 vs. the $25 \mathrm{mM}$ glucose group; ${ }^{\#} p<0.05$ and ${ }^{\#} p<$ 0.01 vs. the $150 \mathrm{mM}$ glucose group

\section{Aloperine inhibited HG-induced RSC96 cell apoptosis.}

The apoptosis of RSC96 cells was significantly induced by HG treatment $(p<0.01)$, while this was reversed by $10 \mu \mathrm{M}(p<0.01)$ and $50 \mu \mathrm{M}(p$ $<0.01$ ) aloperine treatment (Figure 2). These results revealed that aloperine inhibited HGinduced RSC96 cell apoptosis.

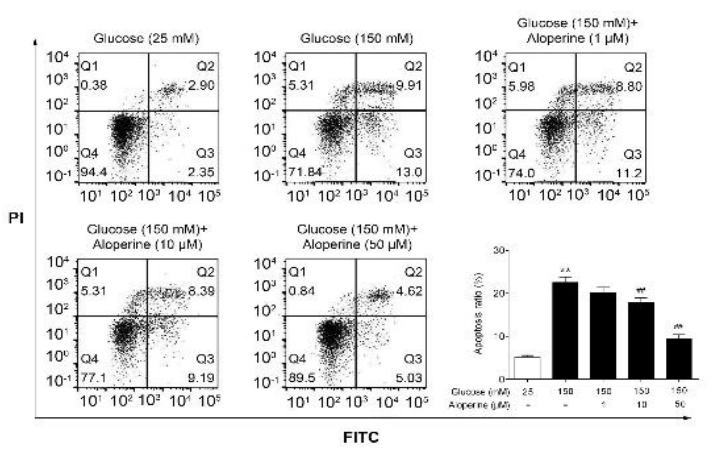

Figure 2: Aloperine inhibited high glucose-induced RSC96 cell apoptosis. Apoptosis was determined using flow cytometry; ${ }^{* *} p<0.01 \mathrm{vs}$. the $25 \mathrm{mM}$ glucose group; ${ }^{\#} p<0.05$ and ${ }^{\#} p<0.01$ vs. the $150 \mathrm{mM}$ glucose group (high glucose)

\section{Aloperine reduced HG-induced oxidative stress in RSC96 cells.}

To further investigate the influence of aloperine on HG-induced Schwann cell injury, oxidative stress was evaluated. The levels of ROS and MDA in RSC96 cells were increased by HG ( $p<$ 0.01 ), which were dose-dependently inhibited by aloperine (all, $p<0.01$, Figure 3 A and B). Moreover, the levels of GST were increased by HG $(p<0.01)$, and were dose-dependently reversed by aloperine ( $p<0.05$; Figure $3 \mathrm{C})$. The level of GPX was decreased in RSC96 cells by HG, and was also dose-dependently reversed by aloperine $(p<0.05$; Figure $3 \mathrm{D})$. Together, these results showed that aloperine remediated HGinduced oxidative stress in RSC96 cells.
A

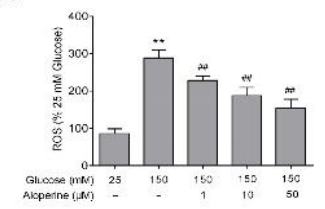

C

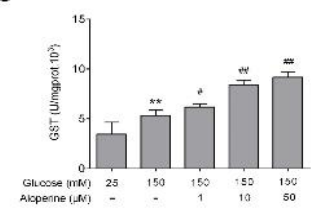

B
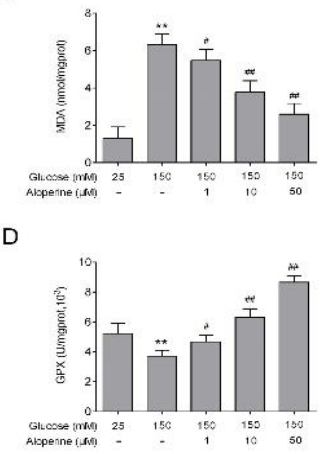

Figure 3: Aloperine reduced the oxidative stress in RSC96 cells caused by high glucose $(150 \mathrm{mM})$. Levels of ROS (A), MDA (B), GST (C), and GPX (D) in RSC96 cells were evaluated using ELISA assays; ${ }^{* *} p<$ 0.01 vs. the $25 \mathrm{mM}$ glucose group; ${ }^{\#} p<0.05$, and ${ }^{\# \#} p$ $<0.01$ vs. the $150 \mathrm{mM}$ glucose group. ROS = reactive oxygen species; MDA = malondialdehyde; GST = glutathione S-transferase; GPX = glutathione peroxidase 
Aloperine increased NRF2/HO-1 pathway activities in RSC96 cells caused by HG.

To determine the underlying mechanism of aloperine on HG-induced cell injury, the levels of NRF2 and HO-1 were determined. The mRNA levels of NRF2 and HO-1 in RSC96 cells were increased by HG treatment (all $p<0.01$ ), and were further increased after treatment with $1 \mu \mathrm{M}$, $10 \mu \mathrm{M}$, and $50 \mu \mathrm{M}$ aloperine (Figure $4 \mathrm{~A}$ and $4 \mathrm{~B}$, $p<0.05$ or $p<0.01$ ). In addition, western blotting results showed that the protein levels of NRF2 and HO-1 were increased in RSC96 cells by HG treatment $(p<0.01)$, and were further increased after treatment with $1 \mu \mathrm{M}, 10 \mu \mathrm{M}$, and $50 \mu \mathrm{M}$ aloperine (Figure $4 \mathrm{C}$ and $4 \mathrm{D}, p<0.05$ or $p<$ $0.01)$. Hence, aloperine activated the NRF2/HO1 pathway in RSC96 cells cultured with HG.
A

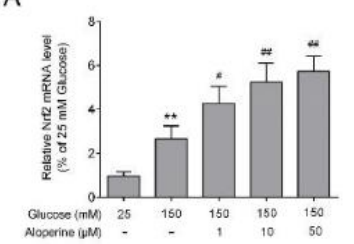

C

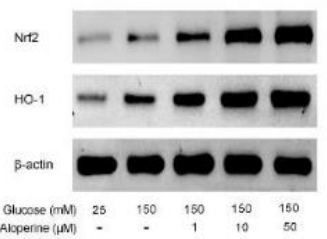

B
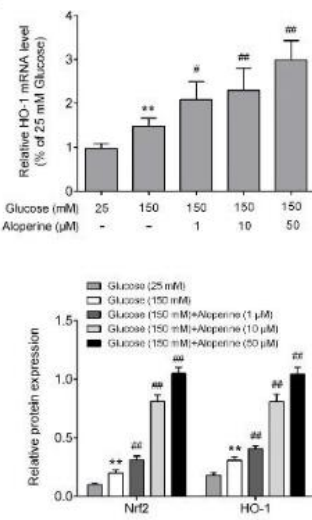

Figure 4: Aloperine activated NRF2/HO-1 pathway in RSC96 cells induced by high glucose $(150 \mathrm{mM})$. The mRNA (A, B) and protein levels (C, D) were determined using qRT-PCR assays or western blotting; * $p<0.01$ vs. the $25 \mathrm{mM}$ glucose group; ${ }^{*} p<$ 0.05 and ${ }^{\#} p<0.01$ vs. $150 \mathrm{mM}$ glucose group. NRF2 = nuclear factor erythroid 2-related factor 2; $\mathrm{HO}-1=$ heme oxygenase-1

\section{DISCUSSION}

Schwann cell injury caused by oxidative stress under long-term hyperglycemia is a critical factor for DPN [5,6]. Hence, it is essential to find effective therapeutic methods to treat DPN.

In our study, the role of aloperine in Schwann cell injury caused by HG was investigated, showing that aloperine increased cell viability and decreased LDH release in RSC96 cells cultured in HG. These results were consistent with previous reports $[19,20]$. A study showed that aloperine elevated cell viability and reduced neuronal injury stimulated by oxygen-glucose deprivation and reperfusion [19]. The increased $\mathrm{LDH}$ release induced by $\mathrm{H}_{2} \mathrm{O}_{2}$ exposure was blocked by aloperine in a cell model of Alzheimer's disease [20]. Likewise, our study revealed that aloperine alleviated RSC96 cell injury induced by HG.

Studies have further revealed that HG leads to apoptosis of Schwann cells [21,22]. The progression of DPN can be alleviated by inhibiting the apoptosis of Schwann cells [22]. Therefore, the protection of aloperine from apoptosis of RSC96 cells caused by HG was determined in this study. The results indicated that increased apoptosis of RSC96 cells caused by HG was dose-dependently reversed by aloperine. A study reported that aloperine decreased the levels of apoptosis-related proteins, Bax and $\mathrm{Bcl}-2$, in pulmonary hypertension induced by monocline [23]. However, aloperine was found to induce cancer cell apoptosis [24]. For example, aloperine inhibited cancer progression by inducing prostate cancer cell apoptosis [24]. This result indicated that the influences of aloperine on cell apoptosis might vary among different cells.

Oxidative stress plays critical roles in the Schwan cell injury induced by HG [7]. The effects of aloperine on oxidative stress were thus investigated. The results showed that the major indicators of oxidative stress, including ROS, MDA, GST, were increased, while GPX was decreased in RSC96 cells after HG treatment, but this was reversed by aloperine. Thus, in Schwan cells aloperine alleviated oxidative stress induced by HG, which was similar to previous studies $[23,25]$. Aloperine attenuated alterations in oxidative stress biomarkers induced by monocrotaline in pulmonary hypertensive rats [23]. Aloperine also reduced oxidation activity induced by $\mathrm{H}_{2} \mathrm{O}_{2}$ [25]. Therefore, these results indicated that aloperine alleviated HG-induced oxidative stress in RSC96 cells.

It is known that NRF2/HO-1 signaling also participates in the regulatory process of Schwan cell injury induced by $\mathrm{HG}$ [11]. Thus, the NRF2/HO-1 pathway might mediate the effect of aloperine on Schwan cell injury caused by HG. In our study, aloperine increased NRF2/HO-1 pathway activity in RSC96 cells induced by HG. In previous studies, aloperine inhibited allergic airway inflammation via activating the NRF2/HO1 pathway [26]. In the subarachnoid hemorrhage model, aloperine activated the NRF2/ARE pathway and ameliorated early brain injury [15].

\section{CONCLUSION}

Aloperine attenuated HG-induced oxidative injury in Schwann cells via activating the NRF2/HO-1 
pathway. Thus, aloperine may be an effective drug for the treatment of DPN.

\section{DECLARATIONS}

\section{Acknowledgement}

This work was supported by the National Natural Science Foundation of China (Grant no. 81574051).

\section{Conflict of interest}

No conflict of interest is associated with this work.

\section{Contribution of authors}

We declare that this work was done by the authors named in this article and all liabilities pertaining to claims relating to the content of this article will be borne by the authors.

\section{Open Access}

This is an Open Access article that uses a funding model which does not charge readers or their institutions for access and distributed under the terms of the Creative Commons Attribution License (http://creativecommons.org/licenses/by/ 4.0) and the Budapest Open Access Initiative (http://www.budapestopenaccessinitiative.org/rea d), which permit unrestricted use, distribution, and reproduction in any medium, provided the original work is properly credited.

\section{REFERENCES}

1. Darivemula S, Nagoor K, Patan SK, Reddy NB, Deepthi CS, Chittooru CS. Prevalence and its associated determinants of Diabetic Peripheral Neuropathy (DPN) in individuals having type-2 diabetes mellitus in Rural South India. Indian J Commun Med 2019; 44(2): 88.

2. Zhang $J$, de Albuquerque Rocha $N$, McCullough PAJRicm. Contribution of ApoCIII to diabetic dyslipidemia and treatment with volanesorsen. Rev Cardiovasc Med 2018; 19(1): 13-19.

3. Veves A, Backonja M, Malik RA. Painful diabetic neuropathy: epidemiology, natural history, early diagnosis, and treatment options. Pain Med 2008; 9(6): 660-674.

4. Kim M, Kim H, Kim D, Kim D, Huh Y, Park C, Chung H-J, Jung J, Jeong NY. Heme oxygenase 1 in Schwann cells regulates peripheral nerve degeneration against oxidative stress. ASN neuro 2019; 11: 1759091419838949.

5. Babizhayev MA, Strokov IA, Nosikov VV, Savel'yeva EL, Sitnikov VF, Yegorov YE, Lankin VZ. The Role of
Oxidative Stress in Diabetic Neuropathy: Generation of Free Radical Species in the Glycation Reaction and Gene Polymorphisms Encoding Antioxidant Enzymes to Genetic Susceptibility to Diabetic Neuropathy in Population of Type I Diabetic Patients. Cell Biochem Biophys 2015; 71(3): 1425-1443.

6. Fang X, Zhang C, Zhang C, Cai Y, Yu Z, Huang Z, Li W, Zhang W. Reactivation of Denervated Schwann Cells by Embryonic Spinal Cord Neurons to Promote Axon Regeneration and Remyelination. Stem Cells Int 2019; 2019

7. Askwith T, Zeng W, Eggo MC, Stevens MJ. Oxidative stress and dysregulation of the taurine transporter in high-glucose-exposed human Schwann cells: implications for pathogenesis of diabetic neuropathy. Am J Physiol Endocrinol Metab 2009; 297(3): E620E628.

8. Zhang H, Liu Y-y, Jiang Q, Li K-r, Zhao Y-x, Cao C, Yao $J$. Salvianolic acid $A$ protects RPE cells against oxidative stress through activation of Nrf2/HO-1 signaling. Free Radical Bio Med 2014; 69: 219-228.

9. Chen P, Li L, Gao Y, Xie Z, Zhang Y, Pan Z, Tu Y, Wang $H$, Han $Q, H u X$. $\beta$-carotene provides neuroprotection after experimental traumatic brain injury via the Nrf2ARE pathway. J Integr Neurosci 2019; 18(2): 153-161.

10. Zhang J, Wang Y, Ji X, Shu Z. Tanshinone IIA protects against dopaminergic neuron degeneration via regulation of DJ-1 and Nrf2/HO-1 pathways in a rodent model of Parkinson's disease. Trop J Pharm Res 2019; 18(5).

11. Yang X, Yao W, Shi H, Liu H, Li Y, Gao Y, Liu R, Xu L. Paeoniflorin protects Schwann cells against high glucose induced oxidative injury by activating Nrf2/ARE pathway and inhibiting apoptosis. I Ethnopharmacol 2016; 185: 361-369.

12. Yu H-I, Shen H-C, Chen S-H, Lim Y-P, Chuang H-H, Tai $T$-S, Kung F-P, Lu C-H, Hou C-Y, Lee Y-R. Autophagy Modulation in Human Thyroid Cancer Cells following Aloperine Treatment. Int J Mol Sci 2019; 20(21): 5315.

13. Yuan $X-Y$, Liu W, Zhang $P$, Wang R-Y, Guo J-Y. Effects and mechanisms of aloperine on 2, 4dinitrofluorobenzene-induced allergic contact dermatitis in BALB/c mice. Eur J Pharmacol 2010; 629(1-3): 147152.

14. Xu YQ, Jin S-J, Liu N, Li Y-X, Zheng J, Ma L, Du J, Zhou $R$, Zhao $C-J$, Niu Y. Aloperine attenuated neuropathic pain induced by chronic constriction injury via antioxidation activity and suppression of the nuclear factor kappa B pathway. Biochem Bioph Res Co 2014; 451(4): 568-573.

15. Song S, Chen $Y$, Han F, Dong M, Xiang $X$, Sui J, Li $Y$, Yang $H$, Liu J. Aloperine activates the Nrf2-ARE pathway when ameliorating early brain injury in a subarachnoid hemorrhage model. Exp Ther Med 2018; 15(4): 3847-3855.

16. Vnukov V, Gutsenko O, Milutina N, Ananyan A, Danilenko A, Panina S, Kornienko I. Influence of SkQ1 on expression of Nrf2 transcription factor gene, ARE-

Trop J Pharm Res, June 2020; 19(6): 1151 
controlled genes of antioxidant enzymes and their activity in rat blood leukocytes. Biochemistry-Moscow 2015; 80(5): 586-591.

17. Tang J, Li L, Li CM, Wu J, Sun Y, Wang GL. Upregulation of HO-1 with Haemin Alleviates LPS-Stimulated Pro-inflammatory Responses Through Downregulation of p38 Signalling Pathways in Rat Liver. Scand $J$ Immunol 2015; 82(5): 443-451.

18. Livak KJ, Schmittgen TD. Analysis of relative gene expression data using real-time quantitative $P C R$ and the 2- $\triangle \triangle C T$ method. Methods 2001; 25(4): 402-408.

19. Ma N-T, Zhou R, Chang R-Y, Hao Y-J, Ma L, Jin S-J, Du $J$, Zheng J, Zhao C-J, Niu Y. Protective effects of aloperine on neonatal rat primary cultured hippocampal neurons injured by oxygen-glucose deprivation and reperfusion. J Nat Med-Tokyo 2015; 69(4): 575-583.

20. Zhao J, Zhang G, Li M, Luo Q, Leng Y, Liu X. Neuroprotective effects of aloperine in an Alzheimer's disease cellular model. Biomedicine \& Pharmacotherapy 2018; 108: 137-143.

21. Sun $L-Q$, Zhao J, Zhang TT, Qu L, Wang X, Xue B, Li XJ, Mu Y-M, Lu J-M. Protective effects of Salvianolic acid $B$ on Schwann cells apoptosis induced by high glucose. Neurochem Res 2012; 37(5): 996-1010.
22. Han J, Tan P, Li Z, Wu Y, Li C, Wang Y, Wang B, Zhao $S$, Liu Y. Fuzi attenuates diabetic neuropathy in rats and protects schwann cells from apoptosis induced by high glucose. PloS One 2014; 9(1).

23. Wu F, Yao W, Yang J, Zhang M, Xu Y, Hao Y, Yan L, Niu $Y$, Sun $T, Y u$ J. Protective effects of aloperin on monocroline-induced pulmonary hypertension via regulation of $R$ ho $A /$ Rho kinsase pathway in rats. Biomed Pharmacother 2017; 95: 1161-1168.

24. Ling Z, Guan $H$, You Z, Wang $C$, Hu L, Zhang L, Wang $Y$, Chen $S$, Xu B, Chen M. Aloperine executes antitumor effects through the induction of apoptosis and cell cycle arrest in prostate cancer in vitro and in vivo. Oncotargets Ther 2018; 11: 2735.

25. Ren D, Ma W, Guo B, Wang S. Aloperine attenuates hydrogen peroxide-induced injury via anti-apoptotic activity and suppression of the nuclear factor-кB signaling pathway. Exp Ther Med 2017; 13(1): 315-320.

26. Wang $C$, Choi $Y H$, Xian Z, Zheng $M$, Piao $H$, Yan $G$. Aloperine suppresses allergic airway inflammation through NF-KB, MAPK, and Nrf2/HO-1 signaling pathways in mice. Int Immunopharmacol 2018; 6: 571579. 